\title{
Characterization of carbon nanotubes produced by arc discharge: Effect of the background pressure
}

\author{
Erik I. Waldorff, Anthony M. Waas, ${ }^{\text {a) }}$ Peretz P. Friedmann, and Michael Keidar \\ Department of Aerospace Engineering, University of Michigan, Ann Arbor, Michigan 48109-2140
}

(Received 25 September 2003; accepted 26 November 2003)

\begin{abstract}
Single walled carbon nanotubes (SWNT) produced by the anodic arc discharge over a range of constant background pressures of helium (100-1000 Torr) were examined under a high-resolution transmission electron microscope, and a Raman spectrometer. It was found that the average SWNT diameter is about $2 \mathrm{~nm}$ and fairly independent of the background pressure. Analysis of the relative purity of SWNTs samples suggests that highest SWNT relative concentration can be obtained at background pressure of about 200-300 Torr. Measured anode ablation rate increases linearly with background pressure. The model of the anodic arc discharge was developed. It was found that the predicted anode ablation rate agrees well with experiment suggesting that electron temperature in the anodic arc is about $0.5 \mathrm{eV}$. (C) 2004 American Institute of Physics. [DOI: 10.1063/1.1642737]
\end{abstract}

\section{INTRODUCTION}

Carbon nanotubes (or just nanotubes) are long tubular chains of carbon molecules. They were discovered in $1991,{ }^{1,2}$ and are produced from graphite carbon similar to the "lead" of a pencil. Nanotubes can be readily created from several different production methods. The three most general methods include production by laser, ${ }^{3-5}$ arc discharge, ${ }^{6-8}$ and chemical vapor deposition (CVD)..$^{9-11}$

Nanotubes are extremely light, remarkably strong yet ductile, and electrically and thermally highly conductive. $^{12-15}$ These favorable physical properties lend themselves to many potential industrial applications; functional nanoscale machinery, microscale electronics, and reinforcement for materials. Nanotubes come in either multiwalled nanotube (MWNT) or single-walled nanotube (SWNT) structures. ${ }^{16}$ Due to sliding between the walls, MWNTs are not a suitable potential composite reinforcement, and therefore SWNTs are preferred as the reinforcing medium for nanotube composites. ${ }^{17,18}$ Hard elastic thin films of pure carbon can be created by depositing carbon nanotubes onto a substrate. ${ }^{19}$

Carbon nanotubes are also among the best emitters; they can operate at a very large current density, as high as 4 $\mathrm{A} / \mathrm{cm}^{2} \cdot{ }^{20}$ In particular vertically aligned nanotubes have superior field emission characteristics due to high aspect ratios, resulting in large field enhancement factors. ${ }^{21,22}$ In this respect a role of the background pressure on the nanotube characteristics produced by CVD was studied. ${ }^{23}$

The mechanism of the formation and growth of the nanotubes in arc discharge is a subject of some controversy in the literature. ${ }^{24}$ Several models of the carbon nanotubes growth were proposed such as the open-ended growth model, ${ }^{25}$ growth from small carbon particles by an electrostatic force, ${ }^{26}$ and the two-step growth model. ${ }^{24}$ In order to better understand the mechanism of carbon nanotubes growth a detailed model of the arc discharge is required. a) Author to whom correspondence should be addressed; electronic mail:
dcw@engin.umich.edu
Previous studies have examined the efficiency of carbon nanotubes as a reinforcing medium in epoxy based shortfiber composites. ${ }^{17}$ The composite modulus for a weight fraction of 5\% was reported to be 3.21-4.21 GPa, i.e., an improvement of $3.6 \%-35.8 \%$ from the pure epoxy modulus. ${ }^{17}$ Other studies have utilized vapor-grown carbon fibers, which include multiwall, single-wall, and carbon fibrils as reinforcement. $^{27}$ This has been done for weight fractions ranging from $5 \%$ to $40 \%$ and $60 \%$, giving a yield strength in the range of 49-69 $\mathrm{MPa}$ (with a steady decline in strength as higher weight fractions are introduced due to interface sliding between matrix and fiber, and fibers inbetween). Furthermore, the elastic modulus was found to increase from $100 \%$ to $350 \%$ for weight fractions of $2 \%$ and $60 \%$, respectively. Ajayan et al. ${ }^{28}$ have produced carbon nanotube composite pellets with a weight fraction of $5 \%$, which revealed a slight increase in composite modulus for the pellets under axial tension and axial compression. However, no means were used to fully disperse the carbon nanotubes within the composite. Stephan et al. ${ }^{29}$ produced SWNTpoly(methylmethacrylate) thin film composites for different weight fractions in order to obtain information about the interaction between the matrix and the SWNTs. Results indicated that homogeneity was not completely obtained, which was due to the fact that the SWNTs used were not pure. Other studies ${ }^{30}$ have also indicated a twofold increase of the modulus for weight fractions of less than $10 \%$ using purified SWNTs.

Even though the arc-discharge method has been widely used in nanotube production, the effect of chamber parameters on the yield and geometric properties (aspect ratio) of nanotubes has not been considered. In particular, several studies $^{6-8}$ have used a range of chamber pressures to produce the nanotubes, yet the effect of a constant chamber pressure on nanotube yield and nanotube aspect ratio have not been investigated. From previous studies, it is known that nanotubes can be produced using various production methods. $^{3-11}$ The range of pressures at which nanotubes can be produced using the arc-discharge method has been determined to be $100-700$ Torr. $^{6-8}$ The pressure range at which 
abundant amounts of carbon nanotubes are produced has been identified to be approximately 500-700 Torr. When producing nanotubes in this pressure range, it has been empirically determined that the yield of nanotubes was a maximum at a pressure of approximately 620 Torr.

The objective of this article is to experimentally study the effect of chamber pressure (held constant during the production) on the arc-discharge production of carbon nanotubes, and the characteristics of the nanotubes produced for nanocomposites reinforcement. The characteristics considered are: quality, quantity, and type of nanotube produced as a function of chamber conditions.

Nanotubes produced over a range of constant pressures were examined under a high resolution transmission electron microscope (HRTEM), to determine their geometric characteristics. Representative samples were further examined using Raman spectroscopy to determine the relative concentration of nanotubes within each sample. When examining the nanotubes under HRTEM, the geometric properties emphasized were the aspect ratio (i.e., ratio of length to diameter of the individual SWNTs). The nanotubes produced by the arcdischarge method are gathered in random bundles, making it difficult to determine the specific lengths of the nanotubes. Thus, since only the diameter of the nanotubes can be determined with the HRTEM, the smaller diameter nanotubes are optimal, since if the length is assumed to be constant a smaller diameter nanotube will lead to a larger aspect ratio.

The experimental details of the arc-discharge method of production are described, followed by an explanation of the preparation and examination procedures for the TEM, Raman spectroscopy, and anode ablation. A model for the anode ablation rate is introduced, with details of the model given in the Appendix. Finally, a discussion of the experimental results and significant conclusions are given.

\section{EXPERIMENTAL SETUP AND PROCEDURES}

The arc-discharge facility consists of a stainless steel flanged chamber that is capped at both ends (with an anode endcap at the bottom and a cathode endcap at the top), with a linear drive connected to the bottom of the chamber acting as the anode feed system (see Fig. 1 for a schematic and photographic representation). Two portholes on the vertical sides of the chamber are connected to a digital pressure transducer and a constant pressure control system (CPCS), respectively. The pressure transducer and the CPCS are furthermore interconnected in order to generate a feedback loop to maintain constant pressure. The generated arc between the anode and cathode is sustained with a constant power supply of $120 \mathrm{~V} \mathrm{dc}$ at $78.50 \mathrm{~A}$, using a LABVIEW feedback program connected to the linear drive of the anode and the power supply generating the arc. The anode and cathode are both pure carbon rods, with the anode being hollow and the cathode being solid. The cathode has a length and diameter of 1.5 and 0.5 in., respectively, while the anode has a length of 3 in., and an outer and inner diameter of 0.25 and 0.125 in., respectively.

The anode is packed with carbon powder and various metal catalysts. Previous quanta sizing and microscope ex-

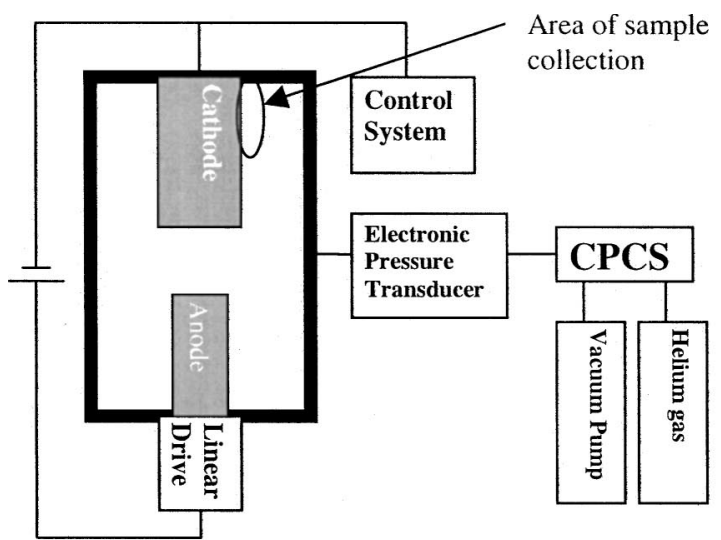

(a)

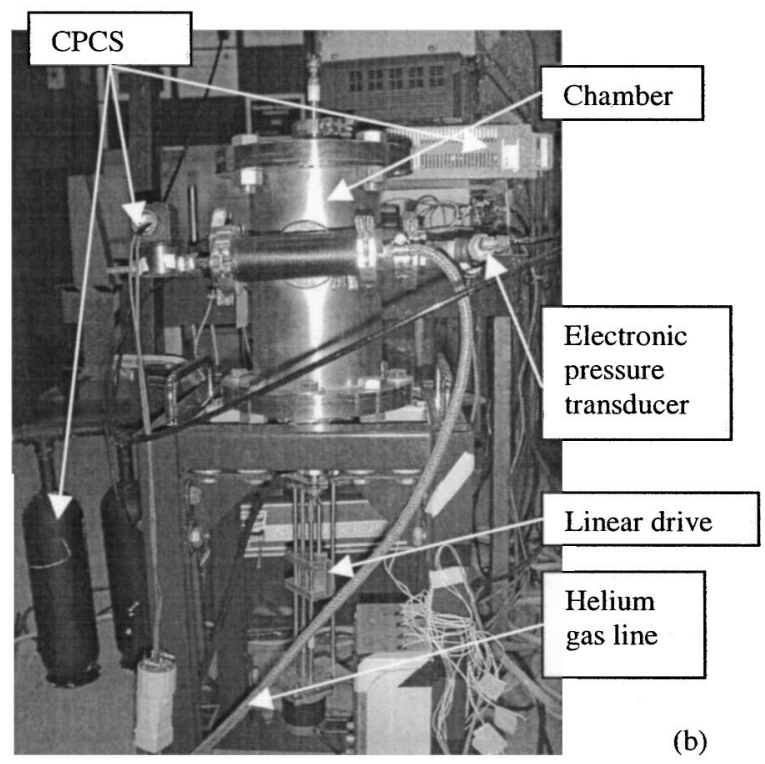

FIG. 1. Schematic (a) and picture (b) of arc-discharge facility.

aminations of arc-discharge products for equal arc runtime has revealed that the catalyst combination yielding the largest amount of nanotubes was $\mathrm{C}: \mathrm{Y}: \mathrm{Ni}$ in a 13:1:4 at. \% ratio. ${ }^{31}$ This ratio was thus utilized for all the carbon nanotube production runs.

Using the production method described above, nanotube samples were produced using constant specific background pressures of helium ranging from 500 to 700 Torr, with 20 Torr increments. Furthermore, samples at 100 and 1000 Torr were produced and examined in order to include extreme pressures. The duration of each production run was $180 \mathrm{~s}$, with the actual production pressure being within a \pm 5 Torr range of the target pressure.

Upon completion of each production run, the arcdischarge facility was cooled to room temperature, and samples were taken from "spiderwebs" of carbon nanotubes hanging from the cathode to the cathode endcap [see Fig. $1(\mathrm{a})]$. The majority $(\sim 95 \%)$ of the carbon nanotubes were deposited on the cathode endcap and the cathode itself as a 1 $\mathrm{mm}$ thick rubber-like layer. The rest $(\sim 5 \%)$ were deposited on the chamber walls as a thin dust layer.

The samples produced were examined under HRTEM using a JEOL-4000EX at the EMAL at the University of 


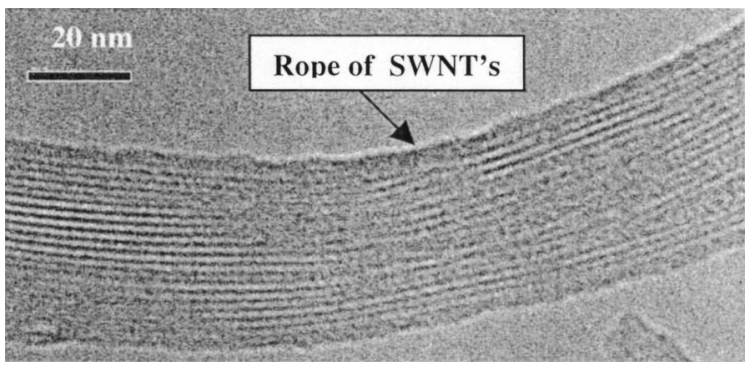

FIG. 2. Typical TEM image of a SWNT rope.

Michigan. The digital TEM images taken of the two samples per specific pressure (i.e., 26 samples total) were postprocessed and the geometry of the nanotubes in the samples were determined. From these examinations a plot of the nanotube diameter versus chamber pressure was produced. During the TEM examinations only SWNT was found in the unpurified samples. A typical TEM image of a SWNT bundle is shown in Fig. 2.

For Raman spectrum analysis, equal portions $(0.02 \mathrm{~g})$ of each sample were suspended in $10.0 \mathrm{~mL}$ of $100 \%$ ethanol, and subsequently ultrasonically mixed for $15 \mathrm{~min}$ in order to generate a homogenous SWNT sample/ethanol liquid. From each SWNT sample/ethanol mix, two drops were placed on a microscope glass slide and left at room temperature for 10 min, during which the ethanol evaporated, leaving a homogeneous sample on the glass. Raman spectrum analyses were performed at three different locations within each of the samples, using a Raman spectrometer. The spectrometer used an argon gas laser $(514.5 \mathrm{~nm})$ running at $80 \mathrm{~A}$ current. The specific run conditions for the Raman spectrometer was a primary slit opening of $150 \mu \mathrm{m}$. The characteristic Raman spectrum for nanotubes has two major peaks for the radial and tangential modes. ${ }^{16}$ The radial mode (RM) is found at a Raman shift of $186 \mathrm{~cm}^{-1}$, and the tangential mode (TM) is found at $1591 \mathrm{~cm}^{-1}$, which was identified and verified for each spot in each sample. Due to difference in intensity amplitude the RM and the TM were examined using scan times of 10 and $1 \mathrm{~s}$, respectively. The power level for the laser was furthermore recorded for each spot in each sample. For each spectrum generated the delta intensity (i.e., the difference between plateau to peak level as shown in Fig. 3) was determined, where the plateau is defined as the constant intensity level away from the peak. The delta intensities were then

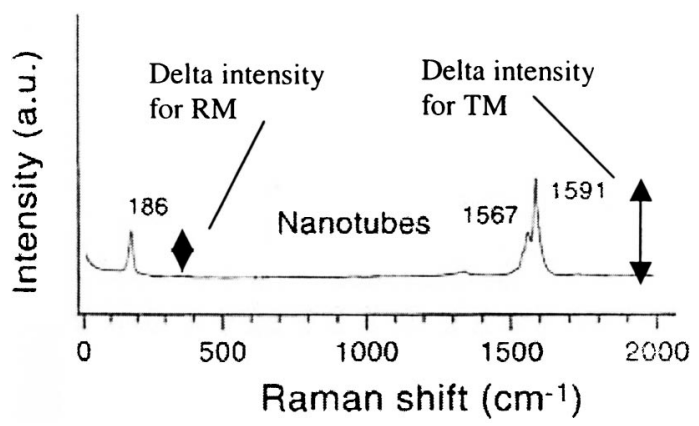

FIG. 3. Typical Raman spectrum for nanotubes with the delta intensity indicated.

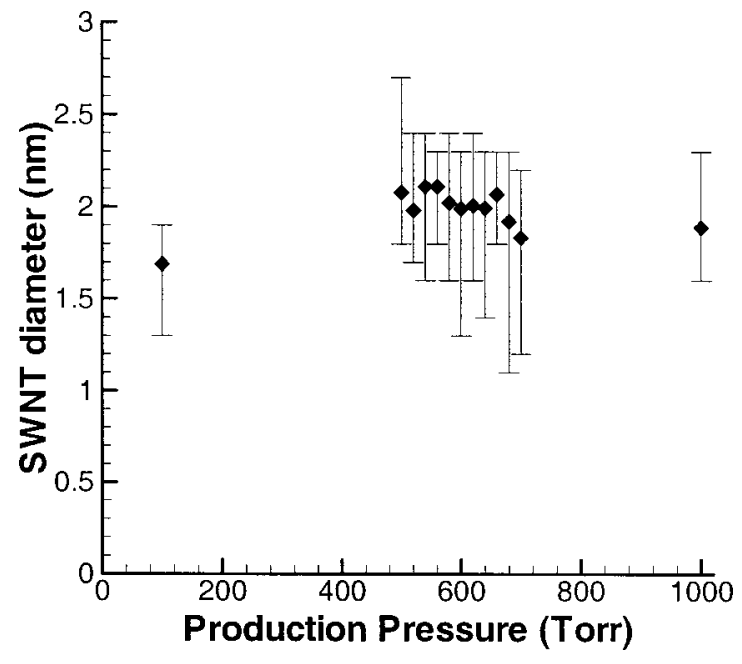

FIG. 4. SWNT diameter (average) vs production pressure.

normalized by dividing through with both the laser power level and the scan time. The average normalized delta intensities for each sample were then computed, and the dependence of the delta intensities or relative concentrations of SWNTs of the samples on the chamber pressure was determined. Chiang et al. ${ }^{32}$ have performed studies of purification of SWNTs, and found that the delta intensity increased as the SWNT sample was carried through several purification processes, i.e., the purity increased as the delta intensity increased. This implies that there is a direct relationship between purity/concentration and the Raman spectrum intensity of the sample. Therefore, this approach was also pursued in the present study. Furthermore, the plateau level ranges for the Raman spectrums were determined to be 80.0-521 a.u. with an average of 175.6 a.u. for the RM, and 42-118 a.u. with an average of 60.8 a.u. for the TM. These low plateau levels indicate that only instrumentation noise was present in the Raman study, thereby rejecting the possibility of background noise being an influence on the delta intensity values.

By measuring the initial and final anode geometry corresponding to each production run, and dividing by the runtime, the average ablation rate of the anode for each production pressure was determined. From these measurements, the dependence of the anode material consumption on the chamber pressure was determined.

\section{RESULTS AND DISCUSSION}

Figure 4 shows the variation of the SWNT diameter as a function of production pressure. Error bars indicate the range of diameters measured through the TEM studies. Individual nanotube diameters do not appear to vary significantly as production pressure is varied. At most there is a slight trend of an increase in diameter as production pressure is increased. This indicates that nanotubes produced by the arcdischarge method in the pressure range 100-1000 Torr are all well suited for applications as reinforcement for structural composites, since such tubes will result in a composite with homogeneous macroscopic composite properties. At present, studies are underway to examine the composite mechanical 


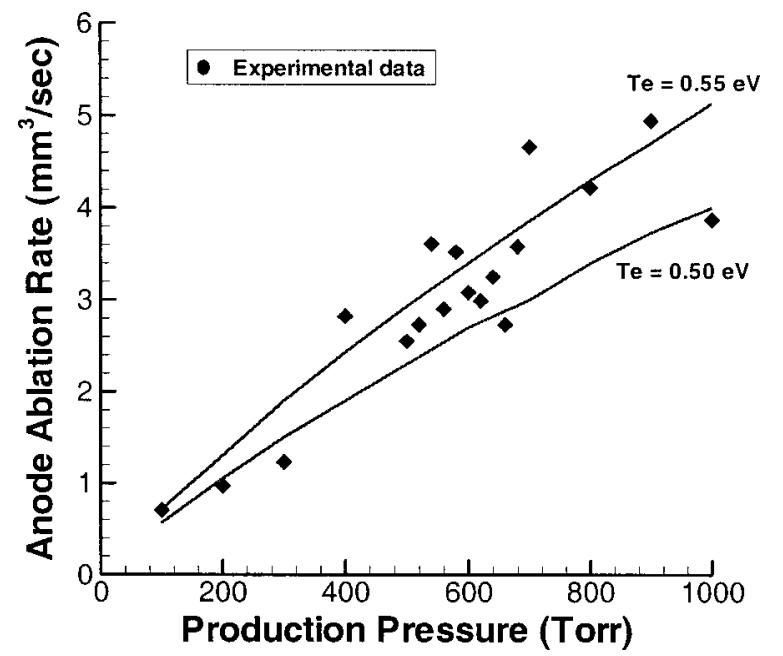

FIG. 5. Anode ablation rate vs production pressure. Comparison between ablation model and experiment.

properties of SWNT reinforced polymer matrix composites, ${ }^{33}$ utilizing mechanics based models of SWNT nanocomposites. ${ }^{14,34-37}$

Experimental results shown in Fig. 5 indicate that as the production pressure increases the ablation rate of the anode increases. Recall that the arc-discharge facility uses helium, which is partially ionized during the arc discharge. As the chamber pressure increases, the heat flux to the anode may increase. This leads to an increase in the surface temperature of the anode, which accelerates the anode ablation rate. To describe these processes, a model of the carbon anode ablation based on a previous kinetic ablation model was developed. ${ }^{38}$ Some details of this model are included in the Appendix.

Using this model (see Fig. 8 for model parameters), the ablation rate was found to be dependent on the production pressure and the plasma temperature $T_{e}$. For the anodic arc it has been determined, experimentally, in previous studies that the plasma temperature ranges from 0.5 to $1.0 \mathrm{eV}^{39-41}$ Knowing this, $T_{e}$ was varied in order to fit the experimental ablation data. As it can be seen from Fig. 5, values of $T_{e}$ between 0.5 and $0.55 \mathrm{eV}$ fit the experimental data quite well. One can see that the model predicts both the ablation rate and its dependence on the background pressure very well.

Figures 6 and 7 show the normalized delta intensity (associated with the Raman spectrum examination) for the RM and TM, respectively, as a function of production pressure. Since the normalized delta intensities are measures of the relative concentrations of nanotubes, the plots indicate the purity level (with respect to the amount of SWNT) of the samples. On the basis of the plots for both the RM and TM we can conclude that the relative concentration of nanotubes in a common pressure range reaches a maximum level between a production pressure of 200 and 300 Torr. These results indicate that nanotube samples produced at this specific pressure are cleaner and thus efforts to purify the postproduction samples will be easier. In addition, if nonpurified nanotubes (nanotubes that are immediately collected from the arc-discharge chamber) are intended for use in compos-

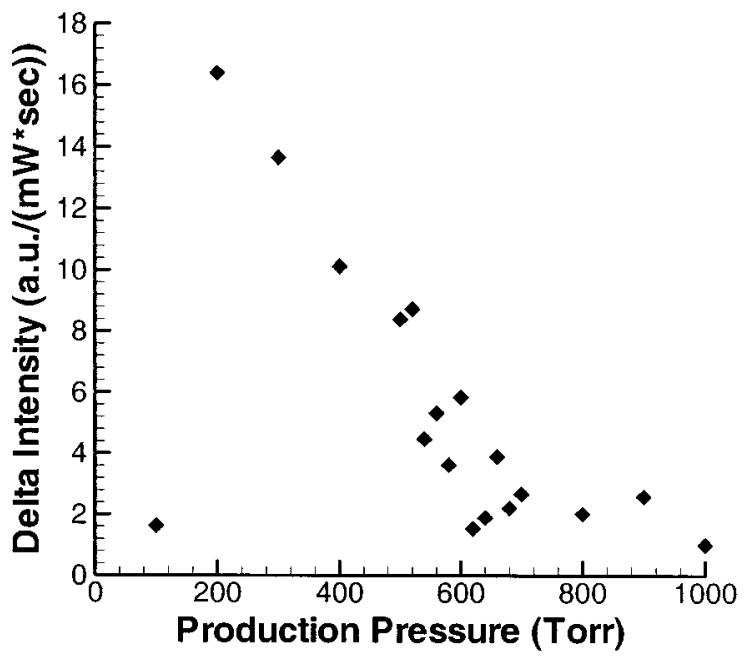

FIG. 6. Raman spectroscopy peak examination of radial mode for SWNT samples.

ites, then samples produced in the range of 200-300 Torr will provide a larger relative SWNT surface area for the matrix to interact with. This in turn will lead to superior mechanical properties of the composite.

\section{CONCLUSIONS}

The quality, quantity, and geometric characteristics of SWNTs produced using the arc-discharge method have been studied over a constant production chamber pressure, ranging from 100 to 1000 Torr. Results from the present study have shown that geometric properties of the SWNTs are constant throughout the examined production pressure range, and that the maximum nanotube concentration can be found for production pressures in the range 200-300 Torr. In addition, since the ablation rate increases with respect to production pressure, one can conclude that the optimal pressure range for SWNT production is between 200 and 300 Torr. This conclusion is valid primarily when emphasizing SWNT production, where characteristics are suitable for application

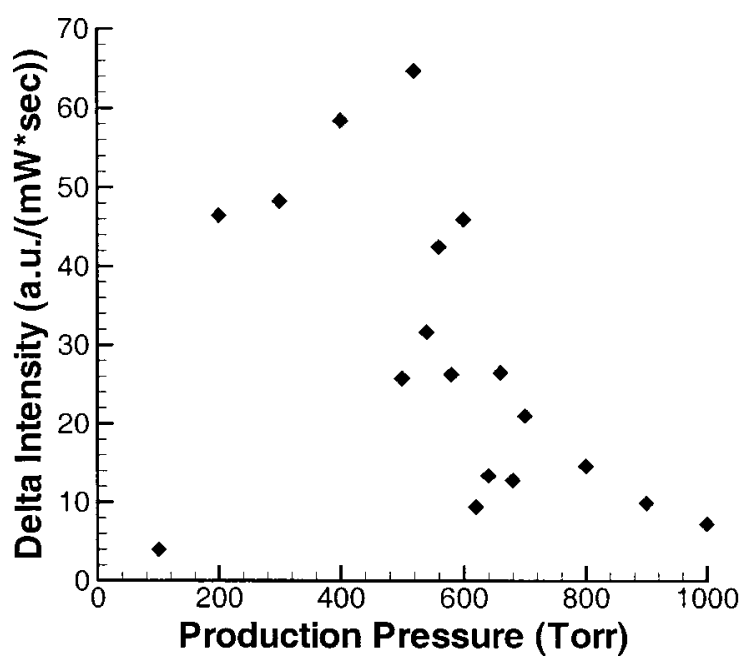

FIG. 7. Raman spectroscopy peak examination of tangential mode for SWNT samples. 


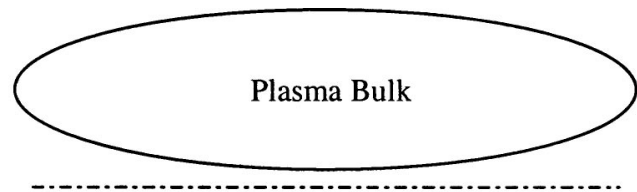

Layer 3

Hydrodynamic Nonequilibrium Layer

Layer 2

Knudsen

Laver

Layer 1

\section{Carbon Anode}

FIG. 8. Schematic representation of layers near ablation surface (Ref. 38).

as reinforcement in a nanocomposite. Furthermore the developed anode ablation model predictions are in good agreement with the experiment.

\section{ACKNOWLEDGMENT}

The initial phase of this study was supported through funds from the NASA Grant No. NAG3-2582 funded by Glenn Research Center. The authors are grateful for this support. E.I.W. would like to thank Professor Roberto Merlin of the Physics Department at the University of Michigan and his student Mariano Trigo for their assistance with the Raman spectroscopy examinations. Kristian Waldorff's time and assistance in building and setting up the arc-discharge facility is gratefully acknowledged. The Raman spectrometer used in the experiment is courtesy of the Materials Examination Laboratory of Professor R. Merlin of the Physics Department, University of Michigan.

\section{APPENDIX: SUMMARY OF ANODE ABLATION MODEL}

In this Appendix, the model of the anode ablation, which is based on the previously developed kinetic model, ${ }^{38}$ is described. The kinetic model takes into account the returned atom flux that forms in the nonequilibrium layer during ablation. This approach makes it possible to calculate the ablation rate for the case when the surface temperature and the density and temperature in the plasma bulk are known. ${ }^{38}$

Two layers are considered (see Fig. 8), the Knudsen layer (a kinetic nonequilibrium layer which is adjusted to the surface that is ablated) and the hydrodynamic nonequilibrium layer, where the electron and heavy particle temperature differ. The right edge of the second layer (see Fig. 8) is assumed to be in thermal equilibrium.

The ablation rate can be calculated as follows:

$$
\Gamma=m n_{1} V_{1},
$$

where $m$ is the carbon atom mass, and $n_{1}$ and $V_{1}$ are density and velocity at the edge of the Knudsen layer, respectively. The kinetic approaches, namely bimodal distribution function and direct simulation Monte Carlo, are used to determine the parameters at the kinetic Knudsen layer edge. ${ }^{38,42,43}$ A coupling solution of the nonequilibrium, Knudsen layer with the hydrodynamic layer, provides a self-consistent so- lution for the ablation rate. Hence by knowing the plasma density, the plasma temperature, and the surface temperature, the ablation rate can be determined. Normally these quantities can be obtained experimentally, but for the present study, a simple model ${ }^{44,45}$ for the plasma is used in order to obtain the desired quantities.

To calculate the carbon anode ablation rate, the ablation model $^{38}$ is combined with the model for electrical discharge presented previously. ${ }^{44,45}$ In the framework of this model, the background pressure determines the particle density in the interelectrode gap. The discharge model is based on the local thermodynamic equilibrium assumption. ${ }^{44,45}$ In this case, the plasma ionization degree (electron density) is determined by knowing the pressure and electron temperature. The electron flux (which is the main energy contributor to the anode) can be calculated using the following equation: ${ }^{39,46}$

$$
Q_{e}=I_{\mathrm{arc}} \cdot\left(2 \cdot T_{e}+U_{a}+U_{w}\right),
$$

where $U_{w}$ is the work function and $U_{a}$ is the anode voltage drop. The anode voltage drop is determined by

$$
U_{a}=T_{e} \cdot \ln \left(\frac{\pi \cdot R_{a}^{2} \cdot e \cdot n_{e} \cdot \sqrt{\frac{k \cdot T_{e}}{m_{e}}}}{I_{\mathrm{arc}}}\right),
$$

where $I_{\text {arc }}$ is the arc current, $R_{a}$ is the anode radius, $n_{e}$ is the electron density, and $m_{e}$ is the electron mass. In this simplified model, it is assumed that power drawn by the anode is dissipated mainly through thermal conductivity. Therefore the balance between energy associated with electron flux and thermal conductivity determines the anode surface temperature.

In order to close the system of equations, the electron temperature should be determined. In this article, the electron temperature is used as a parameter. It is known from numerous experimental and theoretical studies ${ }^{39-41}$ that the electron temperature in the anodic arc discharge is about 0.5-1.0 eV. Therefore in this model the electron temperature is taken to be in this (typical) range.

${ }^{1}$ S. Iijima, Nature (London) 354, 56 (1991).

${ }^{2}$ S. Iijima and T. Ichihashi, Nature (London) 363, 603 (1993).

${ }^{3}$ M. Yudasaka, F. Kokai, K. Takahashi, R. Yamada, N. Sensui, T. Ichihashi, and S. Iijima, J. Phys. Chem. B 103, 3576 (1999).

${ }^{4}$ M. Yudasaka, R. Yamada, N. Sensui, T. Wilkins, T. Ichihashi, and S. Iijima, J. Phys. Chem. B 103, 6224 (1999).

${ }^{5}$ F. Kokai, K. Takahashi, M. Yudasaka, R. Yamada, T. Ichihashi, and S. J. Iijima, J. Phys. Chem. B 103, 4346 (1999).

${ }^{6}$ C-H. Kiang, W. Goddard III, R. Beyers, J. Salem, and D. Bethune, J. Phys. Chem. 98, 6612 (1994).

${ }^{7}$ D. Bethune, C-H. Kiang, M. de Vries, G. Gorman, R. Savoy, J. Vazquez, and R. Beyers, Nature (London) 363, 605 (1993).

${ }^{8}$ S. Aoyama and T. Mieno, Jpn. J. Appl. Phys., Part 2 38, L267 (1999).

${ }^{9}$ Z. Huang, J. Xu, Z. Ren, J. Wang, M. Siegal, and P. Provencio, Appl. Phys. Lett. 73, 3845 (1998).

${ }^{10}$ C. Bower, O. Zhou, W. Zhu, S. Werder, and S. Jin, Appl. Phys. Lett. 77, 2767 (2000).

${ }^{11}$ H. Ago, T. Komatsu, S. Ohshima, Y. Kuriki, and M. Yumura, Appl. Phys. Lett. 77, 79 (2000).

${ }^{12}$ T. Halicioglu, Thin Solid Films 312, 11 (1998).

${ }^{13}$ Y. Jin and F. G. Yuan, in 43rd AIAA/ASME/ASCE/AHS Structures, Structural Dynamics, and Materials Conference, Denver, Colorado (American Institute of Aeronautics and Astronautics, Washington, DC, 2002), AIAA2002-1430. 
${ }^{14}$ G. M. Odegard, T. S. Gates, K. E. Wise, C. Park, and E. J. Siochi, Compos. Sci. Technol., 63, 1671 (2003).

${ }^{15}$ R. S. Ruoff and D. C. Lorents, Symposium on Recent Advances in the Chemistry and Physics of Fullerenes and Related Materials, Reno, Nevada, 1995, p. 143.

${ }^{16}$ M. S. Dresselhaus, M. A. Pimenta, P. C. Eklund, and G. Dresselhaus, Raman Scattering in Fullerenes and Related Carbon-Based Materials (Springer, New York, 2000), p. 314.

${ }^{17}$ L. Schadler, S. Giannaris, and P. Ajayan, Appl. Phys. Lett. 73, 3842 (1998).

${ }^{18}$ R. S. Ruoff, D. Qian, W. K. Liu, W. Ding, X. Chen, and D. Dikin, in 43rd AIAA/ASME/ASCE/AHS Structures, Structural Dynamics, and Materials Conference, Denver, Colorado (American Institute of Aeronautics and Astronautics, Washington DC, 2002), AIAA-2002-1522.

${ }^{19}$ G. A. J. Amaratunga, M. Chhowalla, C. J. Kelly, I. Alexandrou, R. Aharonov, and R. M. Devenish, Nature (London) 383, 321 (1996).

${ }^{20}$ W. Zhu, C. Bower, O. Zhou, G. Kochanski, and S. Jin, Appl. Phys. Lett. 75, 873 (1999).

${ }^{21}$ M. Chhowalla, K. B. K. Teo, C. Ducati, N. L. Rupesinghe, G. A. J. Amaratunga, A. C. Ferrari, D. Roy, J. Robertson, and W. I. Milne, J. Appl. Phys. 90, 5308 (2001)

${ }^{22}$ V. I. Merkulov, D. H. Lowndes, Y. Y. Wei, G. Eres, and E. Voelkl, Appl. Phys. Lett. 76, 3555 (2000)

${ }^{23}$ L. Valentini, J. M. Kenny, L. Lozzy, and S. Santucci, J. Appl. Phys. 92, 6188 (2002).

${ }^{24}$ D. Zhou and L. Chow, J. Appl. Phys. 93, 9972 (2003).

${ }^{25}$ S. Iijima, P. M. Ajayan, and T. Ichihashi, Phys. Rev. Lett. 69, 3100 (1992).

${ }^{26}$ Y. Saito, T. Yoshikawa, M. Inagaki, M. Tomita, and T. Hayashi, Chem. Phys. Lett. 204, 277 (1993).

${ }^{27}$ K. Lozano and E. Barrera, J. Appl. Polym. Sci. 79, 125 (2001).

${ }^{28}$ P. Ajayan, L. Schadler, C. Giannaris, and A. Rubio, Adv. Mater. (Weinheim, Ger.) 12, 750 (2000).

${ }^{29}$ C. Stephan, T. Nguyen, M. Lamy de la Chapelle, S. Lefrant, C. Journet, and P. Bernier, Synth. Met. 108, 139 (2000).
${ }^{30}$ F. Ko, S. Khan, A. Ali, Y. Gogotsi, N. Naguib, G. Yang, C. Li, H. Shimoda, O. Zhou, M. Bronikowski, R. Smalley, and P. Willis, in 43rd AIAA/ ASME/ASCE/AHS Structures, Structural Dynamics, and Materials Conference, Denver, Colorado (American Institute of Aeronautics and Astronautics, Washington DC, 2002), AIAA-2002-1426.

${ }^{31}$ A. Hassanien, M. Tokumoto, Y. Kumazawa, H. Katura, Y. Maniwa, S. Suzuku, and Y. Achiba, Appl. Phys. Lett. 73, 3839 (1998).

${ }^{32}$ I. W. Chiang, B. E. Brinson, R. E. Smalley, J. L. Margrave, and R. H. Hauge, J. Phys. Chem. B 105, 1157 (2001).

${ }^{33}$ E. I. Waldorff, A. M. Waas, and P. P. Friedmann (unpublished).

${ }^{34}$ D. Qian, W. K. Liu, R. S. Ruoff, in 43rd AIAA/ASME/ASCE/AHS Structures, Structural Dynamics, and Materials Conference, Denver, Colorado (American Institute of Aeronautics and Astronautics, Washington DC, 2002), AIAA-2002-1428.

${ }^{35}$ P. Zhang, Y. Huang, P. H. Geubelle, and K. C. Hwang (unpublished).

${ }^{36}$ V. M. Harik, T. S. Gates, and M. P. Nemeth, in 43rd AIAA/ASME/ASCE/ AHS Structures, Structural Dynamics, and Materials Conference, Denver, Colorado (American Institute of Aeronautics and Astronautics, Washington DC, 2002) AIAA-2002-1429.

${ }^{37}$ S. Govindjee and J. Sackman, Solid State Commun. 110, 227 (1999).

${ }^{38}$ M. Keidar, I. D. Boyd, and I. I. Beilis, J. Phys. D 34, 1675 (2001).

${ }^{39}$ I. I. Beilis, R. L. Boxman, and S. Goldsmith, J. Phys. D 32, 128 (1999).

${ }^{40}$ I. I. Beilis, M. Keidar, R. L. Boxman, and S. Goldsmith, Phys. Plasmas 7, 3068 (2000).

${ }^{41}$ H. Ehrich, B. Hasse, M. Mausbach, and K. G. Muller, J. Vac. Sci. Technol. A 8, 2160 (1990).

${ }^{42}$ S. I. Anisimov, Sov. Phys. JETP 27, 182 (1968).

${ }^{43}$ M. Keidar, J. Fan, I. D. Boyd, and I. I. Beilis, J. Appl. Phys. 89, 3095 (2001).

${ }^{44}$ M. Keidar, I. D. Boyd, and I. I. Beilis, IEEE Trans. Plasma Sci. 28, 376 (2000).

${ }^{45}$ M. Keidar, I. D. Boyd, and I. I. Beilis, J. Propul. Power 19, 424 (2003).

${ }^{46}$ A. Lefort, M. J. Parizet, S. E. El-Fassi, and Abbaaoui, J. Phys. D 26, 1239 (1993). 